\title{
Mucormycose du palais chez une patiente ayant contracté le SRAS-CoV-2
}

\author{
Adith Venugopal MSc PhD, Anand Marya BDS MScD
}

Citation : CMAJ 2021 August 16;193:E1255-6. doi : 10.1503/cmaj.211026-f

Voir la version anglaise de l'article ici : www.cmaj.ca/lookup/doi/10.1503/cmaj.211026

$\mathbf{U}$

ne femme de 53 ans ayant un diabète de type $2 \mathrm{mal}$ maîtrisé a consulté aux urgences pour une lésion douloureuse au palais dur. Un mois auparavant, elle avait reçu un résultat positif au test de dépistage du SRAS-CoV-2, mais était asymptomatique et n'avait pas eu besoin de traitement. Huit jours après le diagnostic, la patiente a constaté le développement d'une lésion indolore au palais. Deux semaines plus tard, elle a remarqué que la lésion avait grossi, et elle a commencé à éprouver une douleur intense, qui irradiait du palais, vers le milieu du visage et la mâchoire, accompagnée d'halitose putride. Elle a consulté dans notre hôpital 22 jours après l'apparition de la lésion.

À l'examen, la glycémie à jeun de la patiente était de $9,7 \mathrm{mmol} / \mathrm{L}$ (plage normale 5,6 à 6,9). Nous avons observé une profonde lésion ulcérée au palais dur (figure 1). L'examen histopathologique a révélé de volumineux hyphes non septés présentant de fines cloisons et ramifications (annexe 1, accessible en anglais au www.cmaj. ca/lookup/doi/10.1503/cmaj.211026/tab-related-content). Nous avons identifié Rhizopus microsporum dans les cultures et posé un diagnostic de mucormycose. Notre équipe a procédé au débridement chirurgical de la lésion et prescrit de l'amphotéricine $\mathrm{B}$ liposomique, à prendre à raison de $1 \mathrm{mg} / \mathrm{kg} / \mathrm{j}$ pendant 6 semaines. Nous avons aussi administré à la patiente de l'insuline pour son diabète. Six semaines plus tard, la lésion était disparue et la patiente ne présentait plus de symptômes.

La mucormycose est causée par les champignons saprophytes aérobies Rhizopus, Rhizomucor et Cunninghamella, appartenant à l'ordre des Mucorales' ${ }^{1}$. La maladie se transmet principalement par l'inhalation de spores ou l'inoculation directe sur une lésion de la peau ou d'une muqueuse, à partir de sources présentes dans l'environnement telles que la terre et les déjections d'animaux. La mucormycose peut se manifester par une atteinte rhinocérébrale, pulmonaire, cutanée, gastro-intestinale ou disséminée, et elle peut être fatale. La nécrose tissulaire, causée par une angioinvasion et une thrombose vasculaire, en est une des principales caractéristiques ${ }^{2}$. Le traitement de pre- mière intention est le débridement chirurgical et l'administration d'amphotéricine B liposomique ${ }^{1}$.

La mucormycose s'observe habituellement chez les patients immunodéprimés qui ont un diabète mal maîtrisé ou qui sont sous corticothérapie depuis longtemps, séropositifs pour le VIH ou atteints d'un cancer ou d'insuffisance rénale ${ }^{2}$. Une étude regroupant 101 patients atteints de la COVID-19 et de mucormycose, dont la plupart vivaient en Inde, a révélé que $80 \%$ d'entre eux étaient diabétiques et que $76 \%$ avaient pris des corticostéroïdes pour traiter l'infection au SRAS-CoV- $2^{3}$. Les médecins devraient songer à la mucormycose chez les patients infectés par le SRAS-CoV-2 qui ont une nécrose tissulaire, particulièrement s'ils sont également diabétiques ou ont pris des corticostéroïdes récemment.

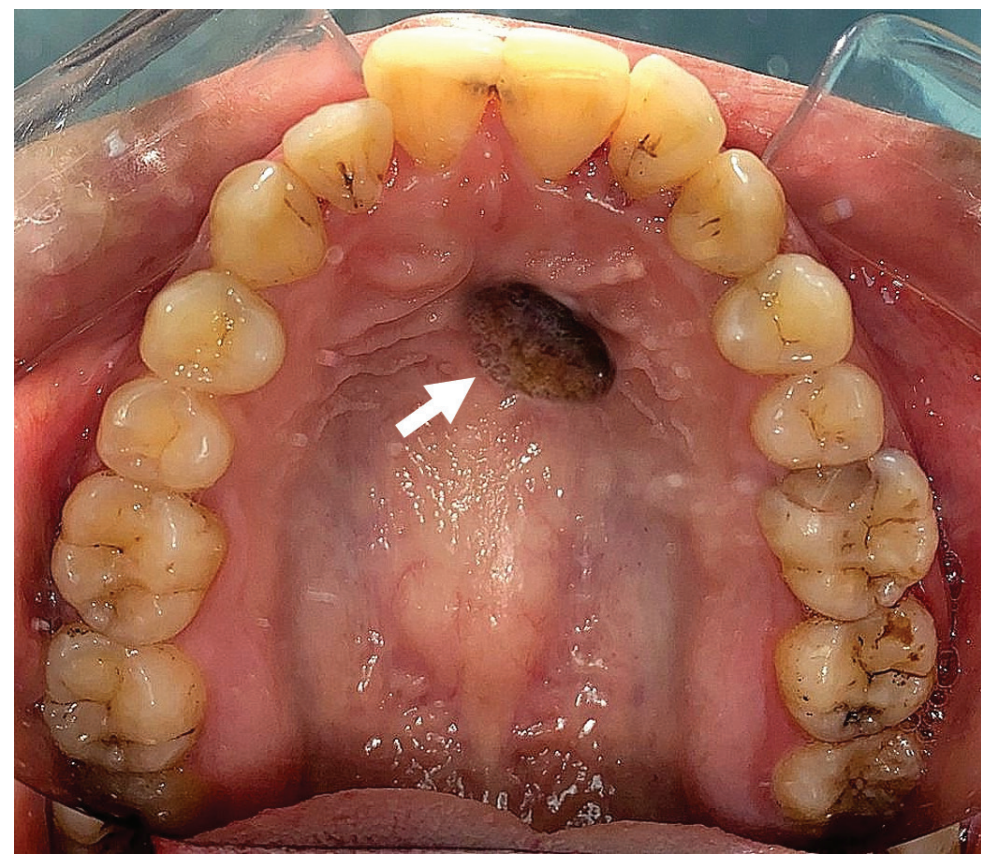

Figure 1 : Photographie montrant un profond ulcère à l'emporte-pièce de $3 \times 2,5$ $\times 0,5 \mathrm{~cm}$ sur la partie médiane du palais dur observé chez une femme de 53 ans. La lésion s'étend de la première à la quatrième crête du palais dur, exposant l'os, et présentes des rebords lobulés et enflés, avec une escarre noirâtre au milieu (flèche). 


\section{Références}

1. Cornely OA, Alastruey-Izquierdo A, Arenz D, et al.; Mucormycosis ECMM MSG Global Guideline Writing Group. Global guideline for the diagnosis and management of mucormycosis: an initiative of the European Confederation of Medical Mycology in cooperation with the Mycoses Study Group Education and Research Consortium. Lancet Infect Dis 2019;19:e405-21.

2. Corzo-León DE, Chora-Hernández LD, Rodríguez-Zulueta AP, et al. Diabetes mellitus as the major risk factor for mucormycosis in Mexico: epidemiology, diagnosis, and outcomes of reported cases. Med Mycol 2018;56:29-43.

3. Singh AK, Singh R, Joshi SR, et al. Mucormycosis in COVID-19: a systematic review of cases reported worldwide and in India. Diabetes Metab Syndr 2021;15:102146.

Intérêts concurrents : Aucun déclaré.

Cet article a été révisé par des pairs.

Les auteurs ont obtenu le consentement de la patiente.

Affiliations : Département d'orthodontie (Venugopal), Saveetha Dental College, Saveetha Institute of Medical and Technical Sciences, Université Saveetha, Chennai, Inde; Département d'orthodontie (Venugopal, Marya), Université de Puthisastra, Phnom Penh, Cambodge.

Propriété intellectuelle du contenu : Il s'agit d'un article en libre accès distribué conformément aux modalités de la licence Creative Commons Attribution (CC BY-NC-ND 4.0), qui permet l'utilisation, la diffusion et la reproduction dans tout médium à la condition que la publication originale soit adéquatement citée, que l'utilisation se fasse à des fins non commerciales (c.-à-d., recherche ou éducation) et qu'aucune modification ni adaptation n'y soit apportée. Voir : https:// creativecommons.org/licenses/by-nc-nd/4.0/deed.fr.

Correspondance : Adith Venugopal, adithvenugopal@saveetha.com; avenugopal@puthisastra.edu.kh 